\title{
Surface Mechanomyogram Reflects Length Changes in Fascicles of Human Skeletal muscles
}

\author{
Yasuhide Yoshitake*, Yasuo Kawakami* ${ }^{* *}$ Hiroaki Kanehisa ${ }^{* * *}$ and Tetsuo Fukunaga ${ }^{* *}$ \\ *Department of Health Sciences, Oita University of Nursing and Health Sciences \\ 2944-9 Megusuno, Oita 870-1201 Japan \\ yoshitake@oita-nhs.ac.jp \\ ${ }^{* *}$ Department of Sports Sciences, WASEDA University \\ 2-579-15 Mikajima, Tokorozawa, Saitama 359-1192 Japan \\ *** Department of Life Sciences (Sports Sciences), University of Tokyo \\ 3-8-1 Komaba, Meguro-ku, Tokyo 153-8902 Japan \\ [Received March 12, 2005 ; Accepted September 7, 2005]
}

\begin{abstract}
To examine the origin of mechanomyogram (MMG), we compared the responses of surface MMG with the changes in the fascicle length determined by ultrasonography during electrically induced contractions of human skeletal muscles. The posterior tibial nerve of subjects $(n=5)$ was stimulated with a stimulus pattern in which the frequency rose up from $1 \mathrm{~Hz}$ to $20 \mathrm{~Hz}$ linearly. The MMG amplitude decreased with increasing stimulation frequency. Reduction of MMG amplitude was in parallel with the decrease in fluctuation of the changes in fascicle length, and these values had a significant positive correlation $(r=0.94, P<0.001)$. The observed direct relation between MMG and architectural change of muscle indicates that surface MMG is generated by the pressure wave produced by the expansion of a number of muscle fibers during contractions.
\end{abstract}

Keywords: mechanomyogram, ultrasonography, architectural change, fascicle length

[International Journal of Sport and Health Science Vol.3, 280-285, 2005]

\section{Introduction}

Mechanomyogram (MMG) is regarded as the recording of mechanical oscillation generated on the surface of muscle concomitant with muscle contraction. Recently, applicable studies have progressed in the fields of physiology, clinical medicine, and rehabilitation (Akataki, et al., 1996, 2002; Barry, et al., 1986; Orizio, et al., 1999; Yoshitake, et al., 2001). From the results of in vitro studies (Barry, 1987; Frangioni, et al., 1987), MMG is assumed to originate in a pressure wave, generated from lateral dimensional expansion when the muscle fiber contracts. No study, however, has been examined the generation mechanism of MMG from architectural change of the muscle.

In studies on human muscles, characteristics of MMG are compared with the contractile properties of activated muscle fibers by microstimulation (Yoshitake, et al., 2002). In a single twitch contraction for instance, MMG has recognized a waveform in muscle fiber contraction/relaxation phases. In repetitive contraction, the fluctuations of tension will be smaller as the stimulation frequency increases and fusion is developed. In this state, architectural change in muscle by muscle fiber contraction/relaxation is lesser, resulting in a smooth wave in muscle tension. Therefore, the pressure wave from contracting muscle naturally becomes smaller with smaller muscle architectural change. Indeed, MMG amplitude and fluctuation of muscle tension decreased simultaneously with an increase of stimulus frequency (Yoshitake, et al., 2002). This supports the hypothesis that the origin of MMG is the pressure wave generated by architectural change (Barry, 1987; Frangioni, et al., 1987). Especially, since the lateral dimensional expansion of the muscle fiber is generated by the change of muscle fiber contraction and relaxation, there should be a close relation between the changes of muscle length and MMG amplitude.

Ultrasonography is a measuring tool that allows real-time measurement of the change of fascicle length accompanying muscle contraction in humans 


\section{Rest}

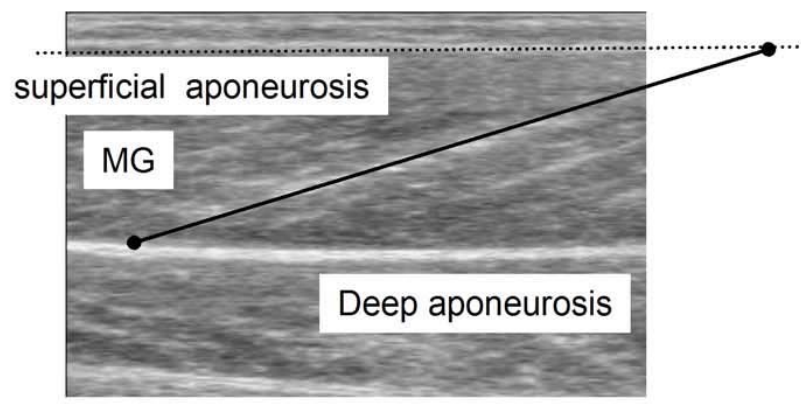

\section{During stimulation}

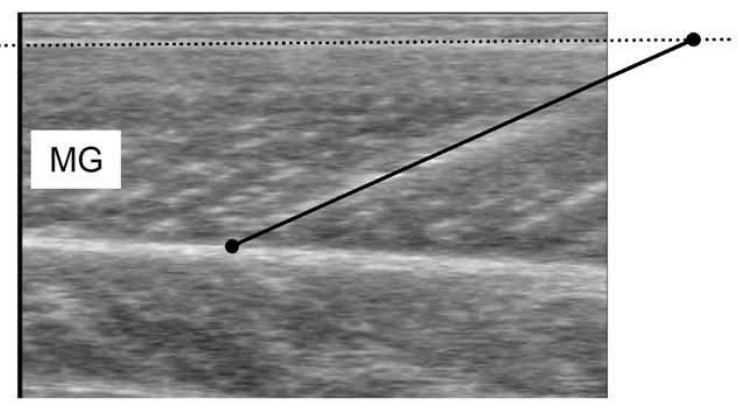

$10 \mathrm{~mm}$

Figure 1 Example of ultrasonic longitudinal images of medial gastrocnemius muscle. Fascicle length was measured as the length of the echo image that runs diagonally from the superficial to the deep aponeurosis along the direction of fascicles.

(Fukunaga, et al., 1997; Kurokawa, et al., 2001). Recent improvements in the measuring technology of ultrasonography have enabled continuous measurement of muscle architectural change of fascicle length shortening and lengthening. The purpose of the present study is to clarify the relation between MMG amplitude and the change of fascicle length during muscle contractions in humans.

\section{Methods}

\subsection{Subjects}

Five healthy men voluntarily participated in this study. The subjects had no medical history or physical signs of neuromuscular disorder. The mean $( \pm \mathrm{SD})$ age, height, and body mass of the subjects were $30 \pm 4.9 \mathrm{yr}, 172.4 \pm 6.4 \mathrm{~cm}$, and $68.3 \pm 7.9 \mathrm{~kg}$, respectively. They gave informed consent according to procedures approved by the Ethics committee of the Faculty of Medicine, The University of Tokyo, Japan.

\subsection{Experimental setup and electrical stimulation}

Each subject was seated on an insulated, straight-back chair with wide belts crossing the chest and abdomen that tightly immobilized the body. An additional strap was used to secure the thigh to the chair. The bottom end of the foot lever plate had a half-round-shaped attachment that surrounded and secured the heel. The heel was also secured with a strap at the bottom end of the foot lever plate. The position of the entire device was carefully adjusted so that the knee was fully extended with the ankle joint angle at $90^{\circ}$.

The posterior tibial nerve was stimulated supramaximally (100- $\mu$ s duration) with a small surface electrode to induce maximal isometric contractions of the triceps surae muscle, in accordance with the method described in a prior study (Yoshitake and Moritani, 1999). We made a stimulation pattern on a DOS/V computer with a digital-to-analogue converter (Trans Era 420, i2net, Japan) which could control the electro-stimulator for inducing contractions. Muscles were tested with a stimulus pattern in which the frequency rose up to $20 \mathrm{~Hz}$ linearly (see Figure 2).

\subsection{Torque and MMG measurements}

Isometric plantarflexion torque evoked by electrical stimulation was measured by an isometric machine (Con-Trex MJ, Bertec, Japan). The method for recording MMG signals was similar to that described in previous studies (Yoshitake, et al., 1999, 2002). The MMG was detected by a piezoelectric microphone (Hewlett Packard 21050A, $0.02-2000 \mathrm{~Hz}$ bandwidth). The microphone was attached with adhesive tape over the belly of the medial gastrocnemius muscle near the probe for ultrasonic image measurement. The MMG signals were band-pass filtered $(1-500 \mathrm{~Hz})$ and amplified (1253A, NEC Medical System, Japan). The MMG and torque signals were stored on a personal computer at a sampling rate of $4 \mathrm{kHz}$ via an analog to digital converter (16-bit, PowerLab/16s, ADInstruments, Australia). 

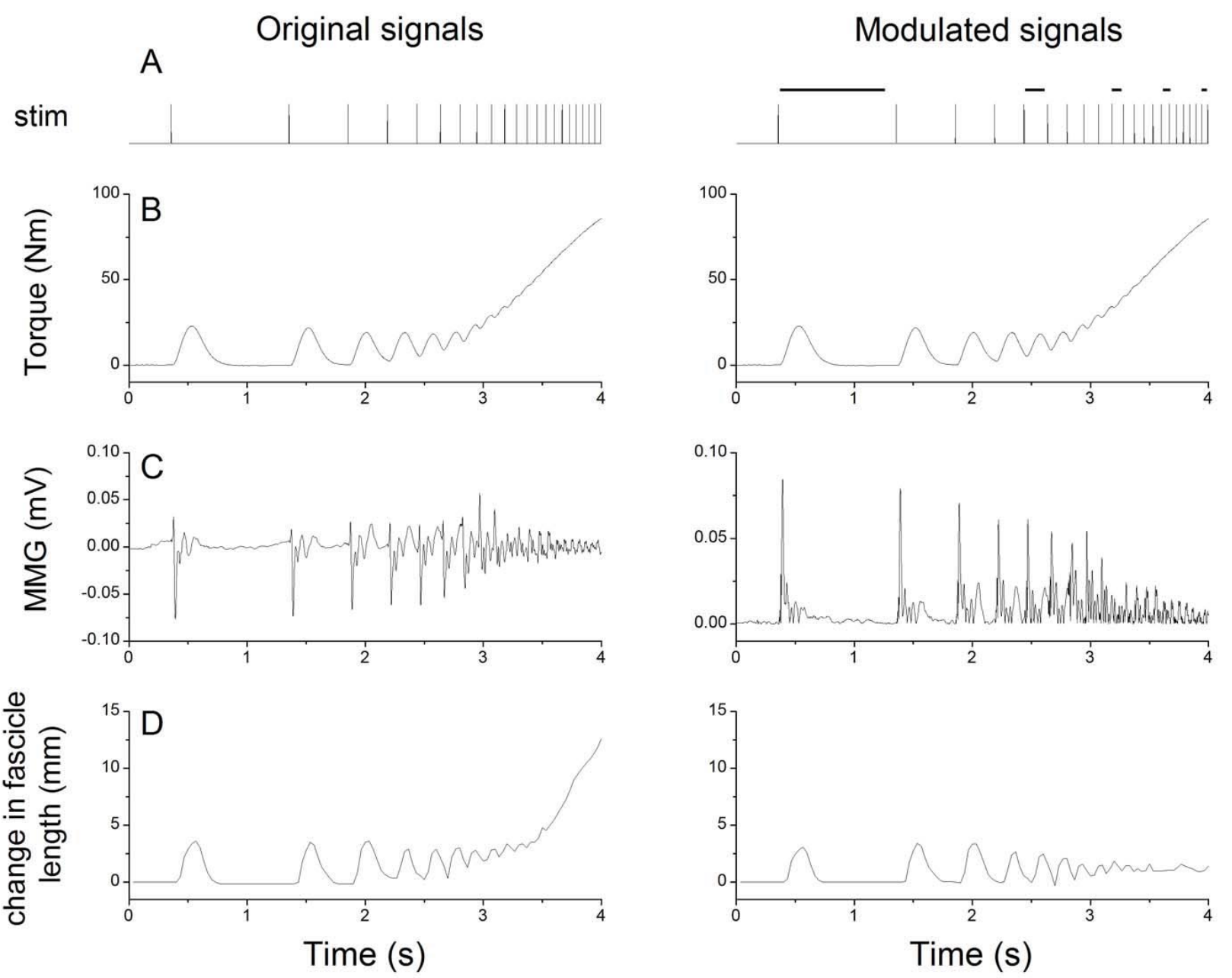

Figure 2 Representative raw signals (left panels) of the stimulation pattern (A), torque (B), MMG (C), and fascicle length change (D) and corresponding modulated signals (right panels). The figure in C-right panel represents the rectified MMG. In D-right panel, fluctuation of fascicle length change is shown. Thick lines indicate the periods for analysis. See detail in text.

\subsection{A real-time brightness mode ultrasonic apparatus}

A real-time brightness mode ultrasonic apparatus (SSD-5500, Aloka, Japan) was used to obtain longitudinal ultrasonic images of the medial gastrocnemius muscle during stimulation. During the measurements, the ultrasound images were recorded on a video tape at $30 \mathrm{~Hz}$ continuously, synchronized with recordings of a clock timer. Fascicle length was measured as the length of the echo image that runs diagonally from the superficial to the deep aponeurosis along the direction of the fascicles (Kurokawa, et al., 2001) (see Figure 1). These analyses were performed using Scion image software (Scion Corporation, USA)

\subsection{Analysis}

To examine the fluctuation of fascicle length when increasing stimulation frequency, the DC component and linear trend of the changes in fascicle length from rest were eliminated by digital filtering to exclude transient responses (Figure 2). Peak-to-peak amplitude of the MMG signals (MMG amplitude) and fluctuations of fascicle length were measured at periods of $1,5,15,20 \mathrm{~Hz}$ stimulation. For further analysis, the obtained MMG amplitude and fluctuations of the fascicle length were then normalized to the respective values at $1 \mathrm{~Hz}$ stimulation frequency.

\subsection{Statistics}

Descriptive statistics were presented as mean and standard error (SE). Statistical analyses were made using 

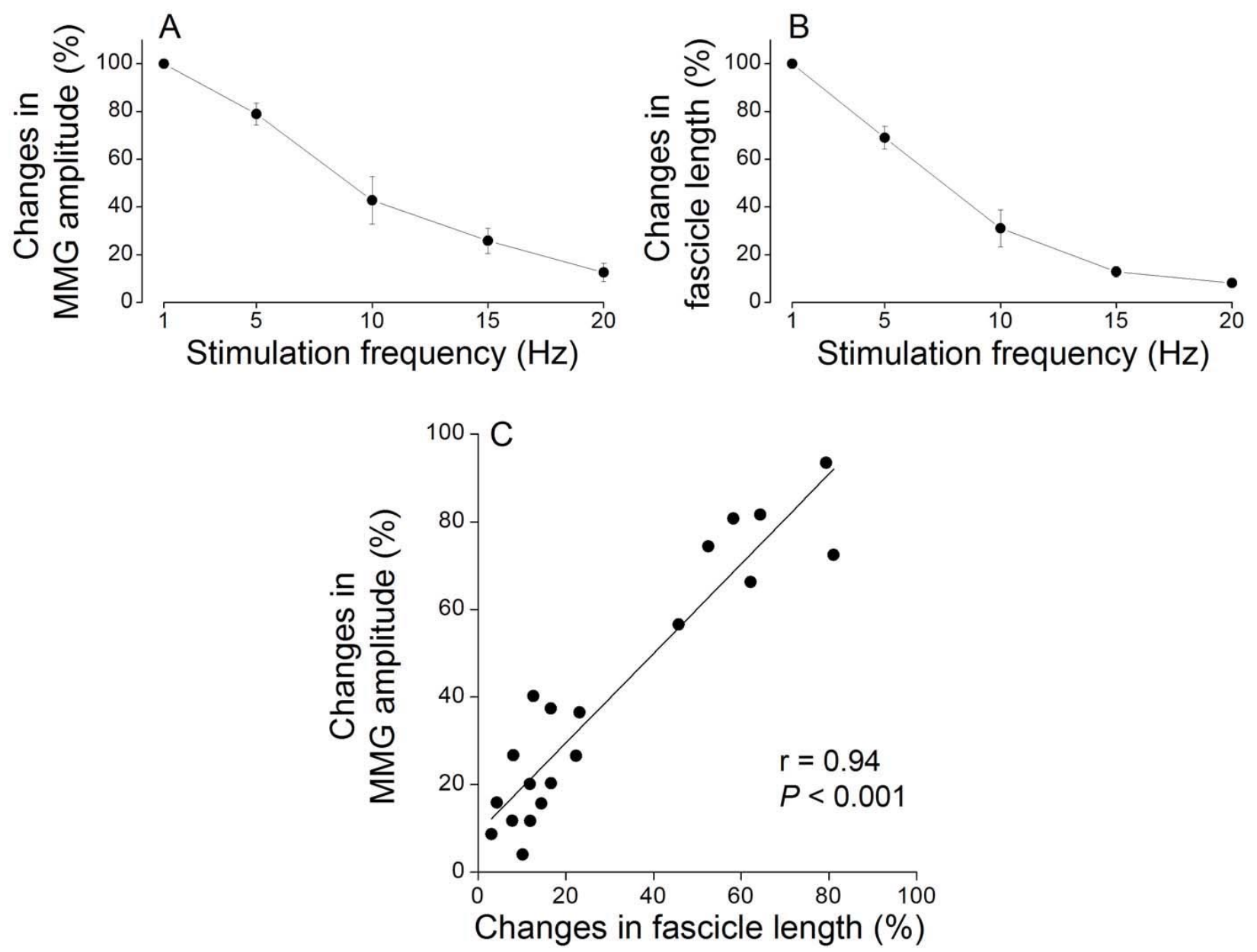

Figure 3 Changes in MMG amplitude (A) and fascicle length (B) at 5, 10, 15, $20 \mathrm{~Hz}$. Data are normalized to the respective values at $1 \mathrm{~Hz}$ stimulation. (C) Correlation between the amplitude of MMG and fascicle length in all subjects by the increase in the stimulation frequency from $1 \mathrm{~Hz}$ to $5,10,15$, and $20 \mathrm{~Hz}$.

linear correlation coefficients (Bravais-Pearson's r). A probability level of $P<0.05$ was considered to be statistically significant.

\section{Results}

In the $1 \mathrm{~Hz}$-stimulated single contraction, the fascicle length sensitively reflects muscle contraction-relaxation phases evidenced by the torque curve (Figure 2). Because the MMG signal arised and converged synchronous with changes in muscle length, MMG should be generated in correspondence to the change of fascicle length.

Figures $\mathbf{3 A}$ and $\mathbf{B}$ show normalized MMG amplitude and fluctuation of fascicle length change with an increase of stimulation frequency, respectively. The fluctuations of both MMG amplitude and changes in fascicle length decreased as stimulus frequency increased. In other words, both MMG amplitude and fluctuation in the fascicle length simultaneously reduced with development of the fusion-process. Figure 3C shows the relation between the change of MMG amplitude and the fluctuations of fascicle length. There was a significant positive correlation between these parameters $(r=0.94, p<0.001)$. These results suggest that MMG amplitude corresponds with fluctuation of fascicle length.

\section{Discussion}

This is the first study to demonstrate the direct relation between MMG characteristics and changes in fascicle length in humans. The main finding of the current study is that decline in amplitude of surface MMG was closely related to the decline in the fluctuations of fascicle length generated by increased frequency of contraction. These results would lead us to support the hypothesis that MMG is caused by the pressure wave generated by architectural change of the muscles. 
In vitro studies (Barry, 1987; Frangioni, et al., 1987), it was speculated that MMG is a pressure wave which occurs when the muscle fiber is dimensionally expanded at contraction. Also, Yoshitake, et al., (2002) demonstrated that MMG amplitude sensitively reflects mechanical activity by muscle contraction/relaxation during microstimulation in human muscle fiber. For example, a pressure wave on the muscle surface is produced by a simple movement of muscle fiber contraction/relaxation during single twitch contraction. In the present study, MMG is also generated and converged in synchronization with muscle fiber contraction and relaxation, respectively (Figure 2). Thus, MMG arises when there is a contraction-induced change in muscular architecture. Hence, these results support the notion that the origin of MMG is a pressure wave generated from lateral dimensional expansion of contracting muscle (Barry, 1987; Frangioni, et al., 1987).

During repetitive stimulations, fusion-process develops with lesser tension fluctuation when stimulus frequency increases, since the next stimulus-induced contraction starts before relaxation of muscle fiber is not completely finished yet. The result of the present study observed decrease of MMG amplitude in parallel with torque fluctuation with increase of stimulus frequency as previous studies (Stokes and Cooper, 1992; Yoshitake et al., 2002) (Figure 3A). Also, lateral expansion of the muscle fiber fluctuates less with increase of stimulus frequency (Frangioni, et al., 1987). In fact, the fluctuation of fascicle length change (the value removing DC component) declined with increasing stimulus frequency (Figure 3B). Further, changes in MMG amplitude showed a strong positive correlation with fluctuation of fascicle length (Figure 3C). These results suggest that MMG amplitude reflects the architectural change of muscle fiber (fluctuation of fascicle length change) and support the hypothesis that the origin of MMG is the pressure wave generated from lateral dimensional expansion of muscle fiber with contraction.

Based on the results of our study on MMG and muscle architectural change during electrically induced muscle contraction, some characteristics of surface MMG during voluntary contractions may reasonably be explained. During voluntary contraction, MMG and electromyogram (EMG) amplitude increase with the increase in exerted force up to moderate intensities because the newly recruited motor unit (MU) contraction creates a rise of fluctuation in expansion of muscle diameter, and in addition the motor unit (MU) discharge rate is not so high as to generate fusion-state (Orizio, et al. 1989). In contrast, at high contraction level, MMG amplitude decreased whereas amplitude of EMG increases continuously due to the additional recruitment of motor units and increase in their discharge rate (Akataki, et al., 2003; Ozirio, et al., 1989; Yoshitake and Moritani, 1999). In high intensity contraction, muscle fiber shortens with increasing discharge rate in motor unit (MU) close to the physiological limit with the tendon elongated to cause a fusion-state (Burke 1981). Thus, the decline in the amplitude of MMG may be attributed to the development of fusion where the high discharge rate of motor units may have greatly reduced the changes in muscle architecture. These notions are further confirmed by the fact that the muscle with greater percentages of "slow" motor units, with lower frequency at which fusion takes place, is more likely to have reduced the amplitude of MMG (Yoshitake and Moritani, 1999).

Lastly, it may be relevant to briefly point out some technical considerations about the employed methods and limitations of the obtained results. The image sampling frequency of $30 \mathrm{~Hz}$ for estimating fascicle length using ultrasonography has a relatively low resolution to examine the changes in the fascile length precisely. Moreover, the directions of lateral expansion estimated from the movement of muscle fiber does not completely correspond with measurement of MMG on the muscle surface because the echograms express two dimensional architectural change. These problems may be solved through the development of measuring equipment in future.

In conclusion, the present study clarified a decline of MMG amplitude with the development of muscle fusion and less fluctuation of the accompanying change in fascicle length. The result suggests that the origin of MMG is the pressure wave caused by architectural change of contracting muscle and that it reflects mechanical oscillation on the muscle surface.

\section{Acknowledgements}

The authors thank Mr. Kentaro Chino, Toshiaki Oda, and Toshiyuki Kurihara (University of Tokyo) for help in this study.

\section{References}

Akataki, K., Mita, K., Itoh, K., Suzuki, N. \& Watakabe, M. (1996). Acoustic and electrical activities during voluntary isometric contraction of biceps brachii muscles in patients with spastic cerebral palsy. Muscle Nerve, 19: 1252-1257. 
Akataki, K., Mita, K., Watakabe, M. \& Ito, K. (2002). Age-related change in motor unit activation strategy in force production: a mechanomyographic investigation. Muscle Nerve, 25: 505-512.

Akataki, K., Mita, K., Watakabe, M. \& Itoh, K. (2003). Mechanomyographic responses during voluntary ramp contractions of the human first dorsal interosseous muscle. Eur J Appl Physiol, 89: 520-525.

Barry, D. (1987). Acoustic signals from frog skeletal muscle. Biophys J, 51: 769-773.

Barry, DT., Leonard, JA., Gitter, AJ., Ball, RD. (1986). Acoustic myography as a control signal for a externally powered prosthesis. Arc Phys Med Rehabil, 67: 267-269

Burke, RE. (1981). Motor units: anatomy, physiology, and functional organization. In J. M. Brookhart, V. B. Mountcastle, V. B. Brooks (eds.), Handbook of Physiology: the nervous system (Vol. II, Motor control). (pp. 345-422). Bethesda, Am Physiol Soc.

Frangioni, J. V., Kwan-Gett, T. S., Dubrunz, L. E. \& McMahon, T. A. (1987). The mechanism of low frequency sound production in muscle. Biophys, 51: 775-783.

Fukunaga, T., Ichinose, Y., Ito, M., Kawakami, Y. \& Fukashiro, S. (1997). Determination of fascicle length and pennation in a contracting human muscle in vivo. J Appl Physiol, 82: 354-358.

Kurokawa, S., Fukunaga, T., Fukashiro, S., Ichinose, Y., Ito, M. \& Kawakami, Y. (2001). Behavior of fascicles and tendinous structures of human gastrocnemius during vertical jumping. J Appl Physiol, 90: 1349-1358.

Orizio, C., Perini, R. \& Veicsteeinas, A. (1989). Muscular sound and force relationship during isometric contraction in man. Eur J Appl Physiol, 58: 528-533.

Orizio C, Baratta RV, Zhou BH, Solomonow M, Veicsteinas A. (1999). Force and surface mechanomyogram relationship in cat gastrocnemius. J Electromyogr Kinesiol, 9: 131-1340.

Stokes, M. J. \& Cooper, R. G. (1992). Muscle sounds during voluntary and stimulated contractions of the human adductor pollicis muscle. J Appl Physiol, 72: 1908-1913.

Yoshitake, Y. \& Moritani, T. (1999). The muscle sound properties of different muscle fiber types during voluntary and electrically induced contractions. J EMG Kinesiol, 9: 209-217.

Yoshitake, Y., Ue, H., Miyazaki, M. \& Moritani, T. (2001). Assessment of lower-back muscle fatigue using electromyography, mechanomyography, and near-infrared spectroscopy. Eur J Appl Physiol, 84: 174-179.

Yoshitake, Y., Shinohara, M., Ue, H. \& Moritani, T. (2002). Characteristics of surface mechanomyogram are dependent on development of fusion of motor units in humans. J Appl Physiol, 93: 1744-1752.

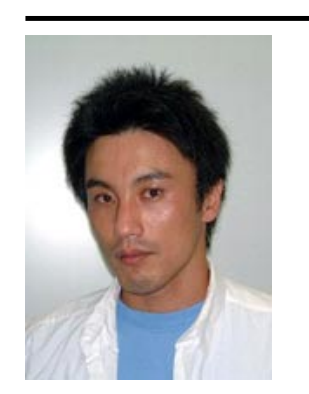

\section{Name: \\ Yasuhide Yoshitake}

\section{Affiliation:}

Department of Health Sciences, Oita University of Nursing and Health Sciences

Address:

2944-9 Megusuno, Oita, Oita 870-1201 Japan

Brief Biographical History:

1996-1999 Graduate School of Human and Environmental Studies, Kyoto University, Kyoto, Japan

1999 Received a Ph.D degree from Kyoto University

1999-2001 Research Associate, Waseda University

2001-2002 Research Fellow of the Japan Society for the Promotion of Science

2002- Research Associate, Oita University of Nursing and Health

Sciences

Main Works:

- Yoshitake, Y., Shinohara, M., Kouzaki, M. and Fukunaga, T. (2004). Fluctuations in plantar flexion force are reduced after prolonged tendon vibration. J Appl Physiol, 97: 2090-2097.

- Yoshitake, Y., Shinohara, M., Ue, H. and Moritani, T. (2002). Characteristics of surface mechanomyogram are dependent on development of fusion of motor units in humans. J Appl Physiol, 93:1744-1752.

\section{Membership in Learned Societies:}

- Japan Society of Exercise and Sports Physiology

- American College of Sports Medicine

- Society for Neuroscience 